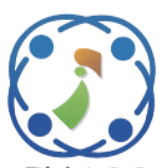

\title{
Dynamic Wireless Power Transfer with a Resonant Frequency for Light Duty Electric Vehicle
}

\author{
Pharida Jeebklum \\ Phumin Kirawanich ${ }^{2}$ \\ Chaiyut Sumpavakup ${ }^{3 *}$ \\ ${ }^{1}$ Power Engineering Technology, College of Industrial Technology, \\ King Mongkut's University of Technology North Bangkok, Bangkok 10800, Thailand \\ ${ }^{2}$ The Cluster of Logistics and Rail Engineering, Faculty of Engineering, Mahidol University, \\ Nakhon Pathom 73170, Thailand \\ ${ }^{3}$ Research Centre for Combustion Technology and Alternative Energy - CTAE \\ and College of Industrial Technology, King Mongkut's University of Technology North Bangkok, \\ Bangkok 10800, Thailand \\ * Corresponding author's Email: chaiyut.s@ cit.kmutnb.ac.th
}

\begin{abstract}
The dynamic wireless power transfer system consists of multiple power transmission sectors in parallel with a single inverter circuit for light duty electric vehicles. The use of multiple power transmission sectors reduces power loss. Usually, each power transmission sector needs to use an inverter for every sector but this system uses one inverter to save cost. This article proposed a study of the dynamic wireless power transfer efficiency between the power transmission sector and the power receiver sector. The performance of the proposed dynamic wireless power transfer system was experimented and compared by varying the gap between the power transmission sector and the power receiver sector, and the speed of the electric vehicle at a resonance frequency. The results show that the dynamic wireless power transfer system had a maximum power transfer efficiency of $82.01 \%$ at a distance between the power transmission sector and the power receiver sector of $0.10 \mathrm{~m}$, with a speed of $0.28 \mathrm{~m} / \mathrm{s}$.
\end{abstract}

Keywords: Dynamic wireless power transfer, Resonance frequency, Light duty electric vehicle.

\section{Introduction}

Electric vehicles are interesting in technology because it can help reduce air pollution problems and energy costs are cheaper than internal combustion engines. The important part that makes electric vehicles still not popular is reliability in the battery over long distances and the long charging time. There are two types of electric charging at present: wired charging and wireless charging. The wired charging may be the cause of electric leakage and can be dangerous to the user while the cable insulation is damaged or cut by a sharp object. The wireless charging is interesting. It is more convenient and safer to use than the wired charging [1]. Wireless charging can be divided into three categories: stationary wireless charging, semi-dynamic wireless charging, and dynamic wireless charging [2].
Stationary wireless charging is wireless power transfer while the electric vehicle is stationary throughout its charge. This limitation means that the charging time is equal to or more than the wired charge. Hence the concept of battery charging while the electric vehicle is moving or dynamic wireless power transfer.

Dynamic wireless power transfer is a way to charge batteries, while electric vehicles are in motion. Multiple power transmission sectors are embedded or placed on the road surface. A power receiver sector is attached to the undercarriage of the vehicle. The electric vehicle moves over the power transmission sectors, the battery can be charged. There are several factors affecting power transfer efficiency. C. Panchal, S. Stegen, and J. Lu [3] studied wireless power transfer technology. They found that the best wireless power transfer performance by using a 
resonant method. The resonance circuit consists of an inductor coil connected to a capacitor in series. The inductor coil is designed to like circular, and there are several series.

In terms of the coil design, Zhang et al. [4] have shown that when the primary coil area is larger than that of the secondary coil, the power transfer efficiency reduces to $50 \%$ with the gap of less than $25 \mathrm{~mm}$. Shuguang et al. [5] studied several selection criteria of the primary coil in terms of the power transfer efficiency. It was seen that the efficiency is inversely proportional with the ratio of the primary to secondary coil areas due to the magnetic flux leakage. Anyapo et al. [6] have shown that the power transfer efficiency depends on the gap between identical primary and secondary coils. The work by Dai et al. [7] has shown that using two primary coils per single secondary coil can enhance the wireless power transfer efficiency nearly four times than that of using a single primary coil.

Concerns about battery life charged while the dynamic wireless power transfer or charged frequently studies found that S. Jeong, Y. J. Jang, D. Kum, and M. S. Lee [8] a frequent battery charge analysis. They found that dynamic wireless battery charging did not shorten battery life faster than usual. It can also reduce the size of the battery to be smaller than the rating, but the battery that is too small may deteriorate faster than normal. Z. Bi, G. A. Keoleian, Z. Lin, M. R. Moore, K. Chen, L. Song, and Z. Zhao [9] studied dynamic wireless battery charging. They found that dynamic wireless battery charging can reduce battery capacity by $21-48 \%$, or one-third to five of the normal battery size. which reduced the weight and price of the vehicle. Battery life estimates are expected to exceed vehicle lifespans of up to 11 years $[10,11]$. The power transfer efficiency depends on the resistance and placement of the inductor coil $[1,7]$.

This paper presents the design of a dynamic wireless power transfer system with a resonance frequency method. The system consists of multiple power transmission sectors in parallel with a single inverter circuit for light duty electric vehicles. The objective was to study and compare the dynamic wireless power transfer efficiency. The parameters for the experiment include the gap varied at $0.10,0.15$, and 0.20 meters, and 3 levels of vehicle speed. The resonant frequency is in accordance with TIR J2954 wireless power transfer standards for PHEVs and EVs for light duty and passenger cars by SAE International [3]. In this paper, a total of five main sections is organized. Section two illustrates the basics of wireless power transfer consisting of wireless power transfer methods, the resonance circuit, and the shape of the inductive coil including compensation topology. Design of a dynamic wireless power transfer system for light duty electric vehicles is described in Section three. Section four gives the experiment. The last section is the result.

\section{Wireless power transfer}

Wireless power transfer is the inductive principle between the power transmission sector and the receiver sector. It like the principle of a transformer but uses air as the core. There are four methods for wireless power transfer [3]. There are capacitive wireless power transfer, magnetic gear wireless power transfer, inductive power transfer, and resonant inductive power transfer. The capacitive wireless power transfer is a method of using a capacitor as a power transfer device. It can be used with low power such as a mobile phone. The power transfer depends on the size of the capacitor and the gap. It is very power transfer efficiency for small gap. The magnetic gear wireless power transfer is a method of using permanent magnets positioned aligned. The electric current flows through the permanent magnet, the permanent magnet of the power transmission sector to rotate and generated torque. It generated inductance with permanent magnets of the power receiver sector causes the transfer power to charge the battery. It considerably challenging for dynamic wireless power transfer. The inductive power transfer is a method of using an inductive coil to transfer power. It is an easy way to change the power and has design flexibility. The resonant inductive wireless power transfer provides more power transfer efficiency than other methods and it is easy to design. It uses a capacitor and an inductive coil is called a resonant circuit.

While the dynamic wireless power transfer system with a single power transmitter can achieve the transfer of power to the running vehicle, a long power transmission medium, e.g., 10-m conductive rail, has high losses, low efficiency, including high installation and maintenance costs [3]. The use of multiple power transmission sections can reduce power loss, directly affecting the power transfer efficiency. This is because the inverter supplies the power to the transmitters only with the presence of the vehicle. That is, two adjacent primary coils are simultaneously supplied by the inverter. The mechanism of using a single inverter also provide effective budget management.

\subsection{Compensation topology of resonant circuit}

The compensation topology different effects on 


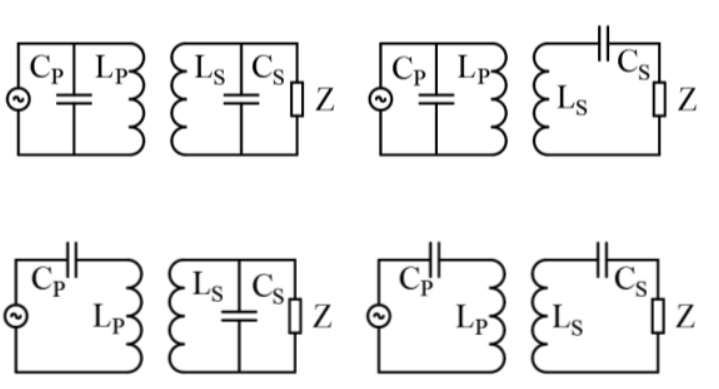

Figure. 1 Compensation topology
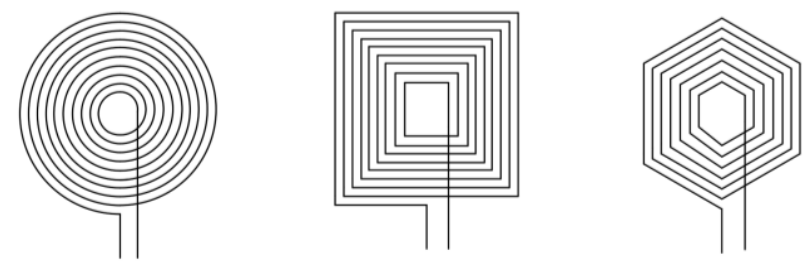

Figure. 2 The shape of the inductor coil

power transfer efficiency. The compensation topology is shown in Fig. 1. The PS and PP are protected so that the primary coil does not operate in the absence of the secondary coil, but the power transfer efficiency is low. The SP can offer high power transfer than the PS and PP, but it is critically dependent on the variation of load. The SS provides the highest power transfer efficiency. It is the most suitable for electric vehicles because the value of the capacitor is independent of the load conditions and mutual inductance. it offers a constant voltage and current for the battery. The inductance values and capacitance values have an error value. This causes the resonance frequency to change which affects the wireless power transfer efficiency. Therefore, there is a way to improve the resonance circuit to be able to the maximum transfer power [12]. The compensation topology for the LCC resonance circuit. It can reduce the loss of power. [1, 13]

\subsection{The shape of the inductive coil}

The shape of the inductor coil affects the magnetic flux in inductance. The shapes are shown in Fig. 2. The rectangular shape creates an eddy current. It increases resistance and heating. It not suitable for high power applications [14]. The center of the hexagonal shape provides high efficiency, but it is reduced at the corner. The oval shape provides more power transfer than other shapes while the secondary coil is misalignment the primary coil. It not suitable for high power. The circular shape has a less eddy current so it can reduce power loss. The primary coil and the secondary coil may be of the same size or different. [3,13]

\subsection{The resonant frequency}

The frequency is high, the reactance of the inductor is high and the reactance of the capacitor is low. It is found that the reactance of the inductor and the capacitor have opposite values. Therefore, if there are frequencies that make the reactance of the inductor equal to the capacitor, the reactance will be completely offset, it has only the resistance value [15, 16]. The frequency that causes such an effect is called the resonance frequency $f$, as shown in Eq. (1) [17]. The standards of the society of automotive engineering determine the resonant frequency used for wireless power transfer is between $81.39-90 \mathrm{kHz}$. $[1,3,18]$

$$
f=\frac{1}{2 \pi \sqrt{L_{P} C_{P}}}=\frac{1}{2 \pi \sqrt{L_{S} C_{S}}}
$$

where $L_{P}$ is the inductance in the primary coil. $C_{P}$ is the capacitance in the primary coil. $L_{S}$ is the inductance in the secondary coil. $C_{S}$ is the capacitance in the secondary coil.

The wireless power transfer circuit is shown in Fig. 3. In the power transmission sector, the voltage $\left(U_{p}\right)$ and the current $\left(I_{p}\right)$. The power of the transmission sector $\left(S_{p}\right)$ can multiply the voltage and current. In the power receiver sector, the voltage $\left(U_{s}\right)$ and the current $\left(I_{s}\right)$. The power of the power receiver $\left(S_{s}\right)$ can multiply the voltage and current. The power transfer efficiency $(\eta)$ can be calculated from in Eq. (2).

$$
\eta=\frac{S_{s}}{S_{p}} x 100
$$

\subsection{The mutual inductance}

The placement of the inductor coil is shown in Fig. 4. $a$ is the radius of the primary coil, $b$ is the radius of the secondary coil, and $z$ is the gap. The mutual inductance $M$, as shown in Eq. (3) [7]. $x$ is position of the secondary coil, $x_{0}$ is position of the secondary coil at $\mathrm{x}=0, x_{I}$ is position of the primary coil set 1 to the secondary coil, and $x_{2}$ is position of the primary coil set 2 to the secondary coil.

$$
M=\sum_{i=1}^{i=n_{p}} \sum_{j=1}^{j=n_{s}} M_{i j}
$$

where 


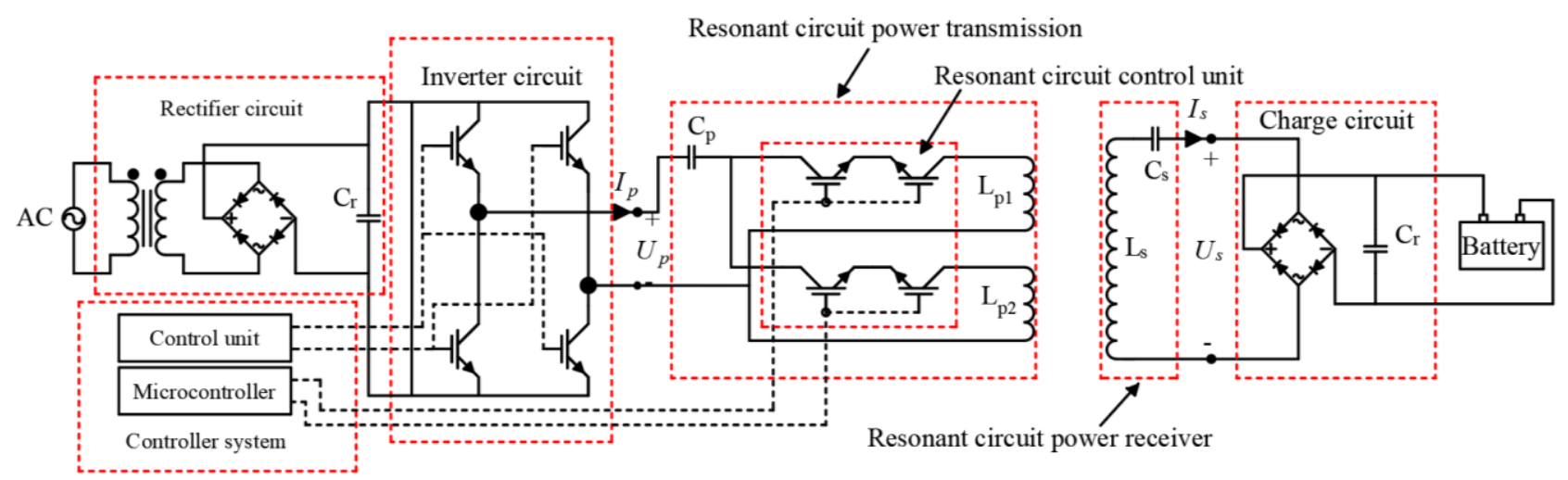

Figure. 3 Wireless power transfer circuit

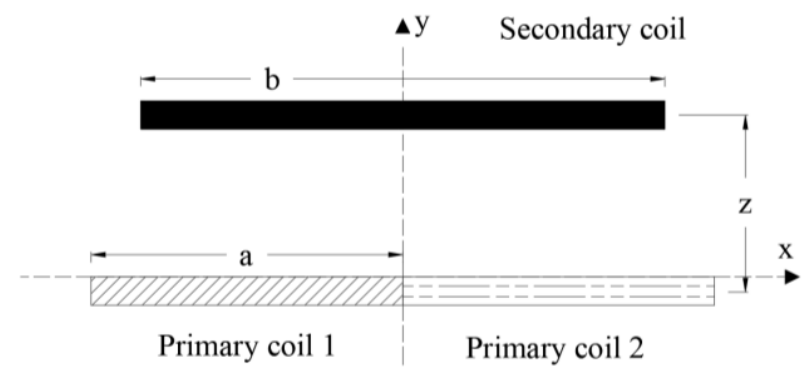

Figure. 4 Placement of the inductor coil

$$
\begin{aligned}
& M_{i j}=\frac{\mu_{0} \pi a^{2} b^{2}}{2\left(a^{2}+b^{2}+z^{2}+x^{2}\right)^{\frac{3}{2}}}\left[1-\frac{3}{2} \delta\right. \\
& +\frac{15}{32} \xi^{2}\left(1-\frac{21}{2} \delta\right) \\
& +\frac{15}{16}\left(\lambda^{2}+\phi^{2}\right)\left(1-\frac{7}{4} \delta\right) \\
& \delta=\frac{x^{2}}{a^{2}+b^{2}+z^{2}+x^{2}} \\
& \xi=\frac{2 a b}{a^{2}+b^{2}+z^{2}+x^{2}} \\
& \lambda=\frac{2 x a}{a^{2}+b^{2}+z^{2}+x^{2}} \\
& \phi=\frac{2 x b}{a^{2}+b^{2}+z^{2}+x^{2}} \\
& x_{1}=-a-x_{0} \quad ; x_{0}<-a \\
& x_{1}=a+x_{0} \quad ; x_{0}>-a \\
& x_{2}=a-x_{0} \quad ; x_{0}<a \\
& x_{2}=x_{0}-a \quad ; x_{0}>a
\end{aligned}
$$

\section{Design of a dynamic wireless power transfer system}

The dynamic wireless power transfer system is shown in Fig. 5, It can charge a battery size $48 \mathrm{~V} 10$
Ah and It can be applied to small electric vehicles. The system consists of two main parts which are the power transmission sector and the power receiver sector.

\subsection{The power transmission sector}

The power supply is $24 \mathrm{~V} 50 \mathrm{~Hz}$. The rectifier circuit converts AC voltage to DC voltage. The inverter circuit generates a resonant frequency. The resonant circuit consists of an inductive coil and a capacitor in series. The inductive coils are a circular shape. The inductance of the primary coil is $80 \mu \mathrm{H}$ and the diameter is 0.20 meters. The power transmission sector uses 10 coils so the distance of the primary coils length 2 meters to have a symmetrical inductor coil placement distance. In the experiment using the inductor coil of the power transmission sector 2 coils at a time so the inductance of the power transmission sector at a time is $40 \mu \mathrm{H}$. The capacitance of the capacitor is $88 \mathrm{nF}$. The resonance frequency is $84.829 \mathrm{kHz}$.

The control system is selected the primary coil which selects only the set under the secondary coil to reduce power loss. The light dependent resistors (LDR) are sensing devices the position of the electric vehicle from the laser light, which is installed at the bottom of the electric vehicle. LDR is installed on any primary coil and sends data to the microcontroller for selects the resonant circuit control unit to supply power.

\subsection{The power receiver sector}

The resonant circuit consists of an inductive coil and a capacitor in series. The inductive coils are a circular shape. The small electric vehicles have a width of the undercarriage that does not exceed 1.30 meters so the diameter of the inductor coil of the power receiver sector should not exceed the width of the electric vehicle suspension. The inductance of the inductor coil is $2240 \mu \mathrm{H}$ and the diameter is 0.37 


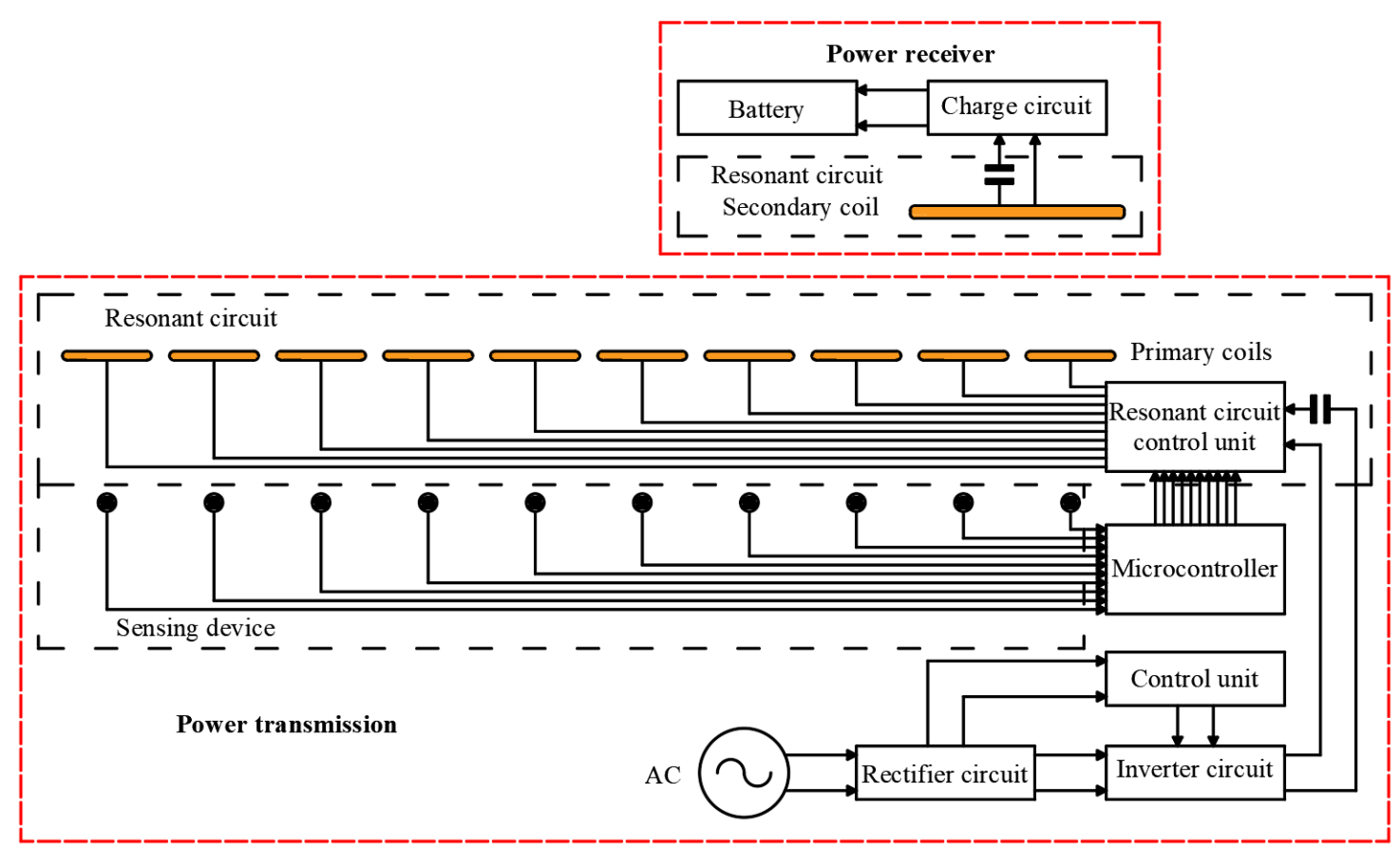

Figure. 5 dynamic wireless power transfer system

Table 1. Parameters of the system

\begin{tabular}{|c|c|}
\hline Parameters & Values \\
\hline$L_{P 1} / L_{P 2}$ & $80 \mu \mathrm{H}$ \\
\hline$C_{P}$ & $88 \mathrm{nF}$ \\
\hline$L_{S}$ & $2240 \mu \mathrm{H}$ \\
\hline$C_{S}$ & $1.57 \mathrm{nF}$ \\
\hline$f$ & $84.829 \mathrm{kHz}$ \\
\hline$a$ & $0.20 \mathrm{~m}$ \\
\hline$b$ & $0.37 \mathrm{~m}$ \\
\hline$z$ & $0.10-0.20 \mathrm{~m}$ \\
\hline
\end{tabular}

Table 2. Mutual inductances

\begin{tabular}{|c|c|c|c|c|}
\hline \multirow{2}{*}{$\begin{array}{c}\text { Gap } \\
(\mathbf{m})\end{array}$} & \multicolumn{4}{|c|}{ Mutual inductance (mH) } \\
\cline { 2 - 5 } & Maximum & Mean & Minimum & Midpoint \\
\hline 0.10 & 0.2100 & 0.1035 & -0.0079 & 0.1137 \\
\hline 0.15 & 0.1450 & 0.0800 & -0.0011 & 0.0925 \\
\hline 0.20 & 0.0982 & 0.0598 & 0.0042 & 0.0703 \\
\hline
\end{tabular}

meters. The capacitance of the capacitor is $1.57 \mathrm{nF}$. The parameters of the system can be summarized as shown in Table 1.

The charger is converting high-frequency $\mathrm{AC}$ voltage to DC voltage for a battery rated $48 \mathrm{~V} 10 \mathrm{Ah}$. The electric motor used to drive electric vehicles is $48 \mathrm{~V} 550 \mathrm{~W}$.

The mutual inductance of the system is calculated from Eq. (3) as shown in Fig. 6. A small gap gives a large mutual inductance. The maximum mutual inductances are position the secondary coil to move closer to the midpoint of the primary coil. The midpoint is the secondary coil between the two primary coils which gives the same mutual inductance. The minimum mutual inductances are position the secondary coil to move far to the primary coil. The mutual inductance can be summarized as shown in Table 2.

\section{Results and discussion}

The experimental set-up is detailed in Fig. 7. At the primary side, the rectifier circuit provide the ACDC voltage conversion. The power transmission sector consists of 10 primary coils. The inverter circuit generates the waveform with a resonant frequency of $84.829 \mathrm{kHz}$. The resonant circuit at the secondary side consists of an inductive coil and a capacitor in series.

In the experiment, the power is transferred through 2 primary coils simultaneously. The control system unit selects the set of primary coils that match the presence of the secondary coil through the detection of LDR sensor attached with the electric vehicle. The charger finally converts high-frequency AC voltage to DC energy for a battery storage. The experiment is carried out through a variation of the gap at $0.10,0.15$, and 0.20 meters and the movement speed of electric vehicles at 3 levels, i.e., level 1 at $0.17 \mathrm{~m} / \mathrm{s}$, level 2 at $0.28 \mathrm{~m} / \mathrm{s}$, and level 3 at $0.56 \mathrm{~m} / \mathrm{s}$. The resonant frequency waveform was measured at the resonance circuit power transmission sector as shown in Fig.8, channels 1 and 2 are voltage and current waveforms, respectively. 


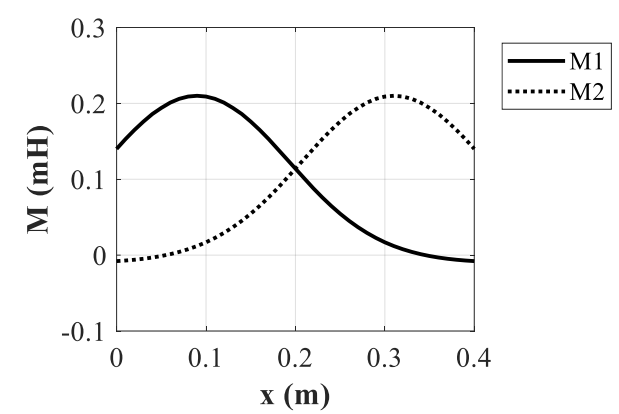

(a)

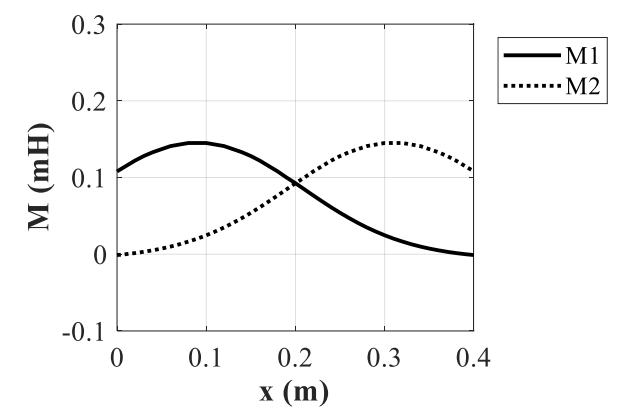

(b)

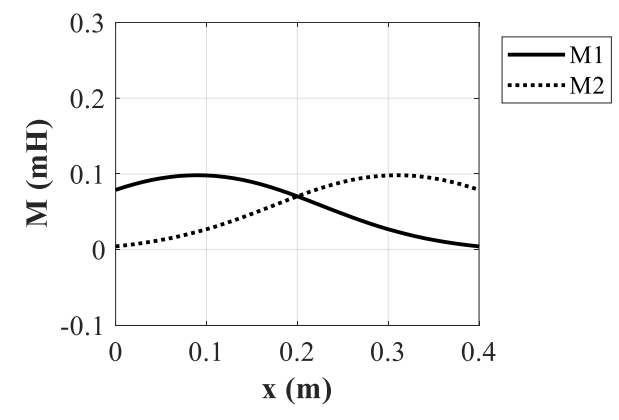

(c)

Figure. 6 Mutual inductances: (a) Gap $0.10 \mathrm{~m}$, (b) Gap $0.15 \mathrm{~m}$ and (c) Gap $0.20 \mathrm{~m}$

\subsection{Comparison results in case of adjusting the gap}

\subsubsection{The gap is $0.10 \mathrm{~m}$}

Comparison of dynamic wireless power transfer efficiency at gap 0.10 meter when the speed changes are shown in Fig. 9, it was found that the speed level 2 gave the highest power transfer efficiency $82.01 \%$ and an average power transfer efficiency of $64.62 \%$. Speed level 2 gave more power transfer efficiency than speed level 1 due to the power receiver at the end of the experiment of speed level 2 increased. At speed level 3 is the fastest speed. The power transfer efficiency is lower than that of the other speed levels because it takes a short time to move over each inductor coil of the power transmission sectors. The power receiver sector may not be able to receive a lot

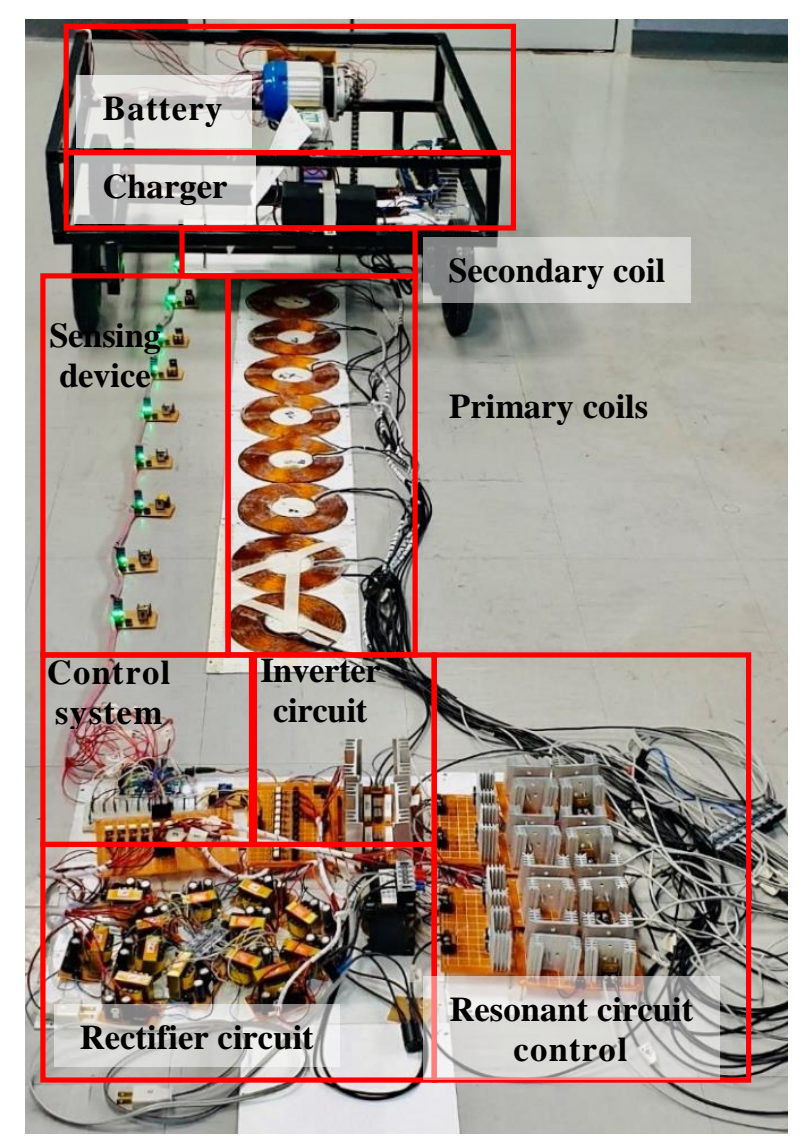

Figure. 7 Experimental set-up

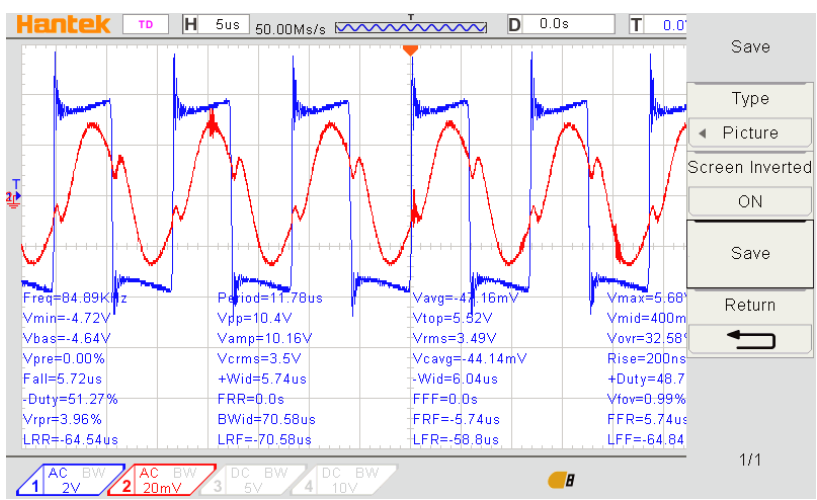

Figure. 8 Resonant frequency waveforms

of power, but the power transfer efficiency is smoother resulting in more continuous efficiency than other speed levels. At all speed levels, the power transfer efficiency is smoother resulting in similar power transfer efficiency at the beginning of the experiment, but at the end of the experiment the power transfer efficiency was lower due to the power supply system to 2 sets of power transmission coils, when the power receiving sector moves over. It can receive power only 1 set thus reducing the power transfer efficiency. Although the power transfer efficiency when the speed level changes are different. They are still considered similar so indicating that the 


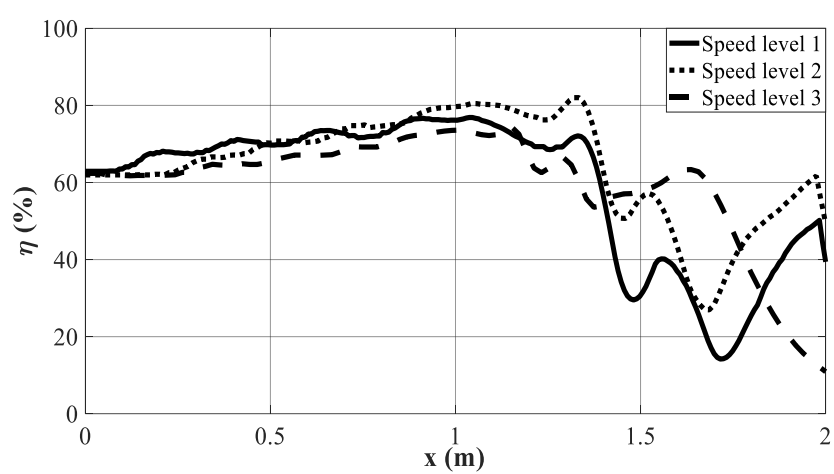

Figure. 9 Power transfer efficiency at gap $0.10 \mathrm{~m}$

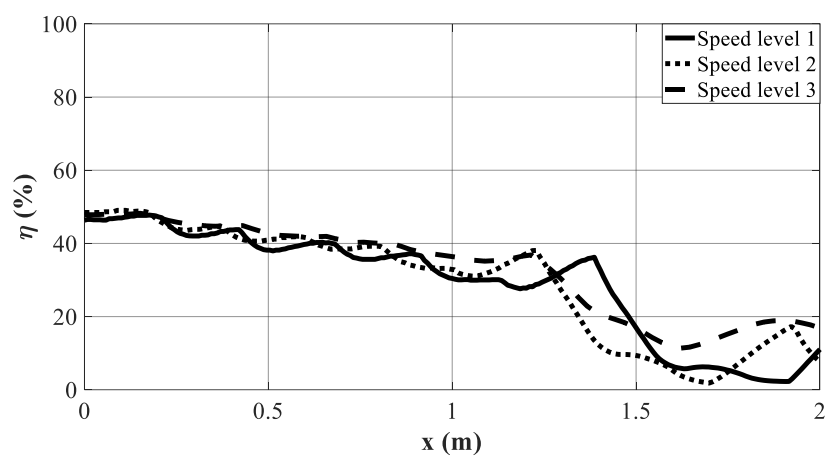

Figure. 10 Power transfer efficiency at gap $0.15 \mathrm{~m}$

speed has a low impact on the power transfer efficiency.

\subsubsection{The gap is $0.15 \mathrm{~m}$}

Comparison of dynamic wireless power transfer efficiency at gap 0.15 meter when the speed changes are shown in Fig. 10, it was found that the speed level 2 gave the highest power transfer efficiency $49.03 \%$. The power transfer efficiency value has a very different. It makes the average power transfer efficiency lower than the speed level 3. The speed level 3 has an average power transfer efficiency of $32.92 \%$. At the speed level 3 is the fastest speed so the power transfer efficiency is smoother, resulting in more continuity than other speed ratings and more average power transfer efficiency. At all speed levels, the power transfer efficiency is smoother resulting in similar power transfer efficiency at the beginning of the experiment, but at the end of the experiment the power transfer efficiency was lower due to the power supply system to 2 sets of power transmission coils, when the power receiving sector moves over. It can receive power only 1 set thus reducing the power transfer efficiency. Although the power transfer efficiency when the speed level changes are different. They are still considered similar so indicating that the speed has a low impact on the power transfer efficiency.

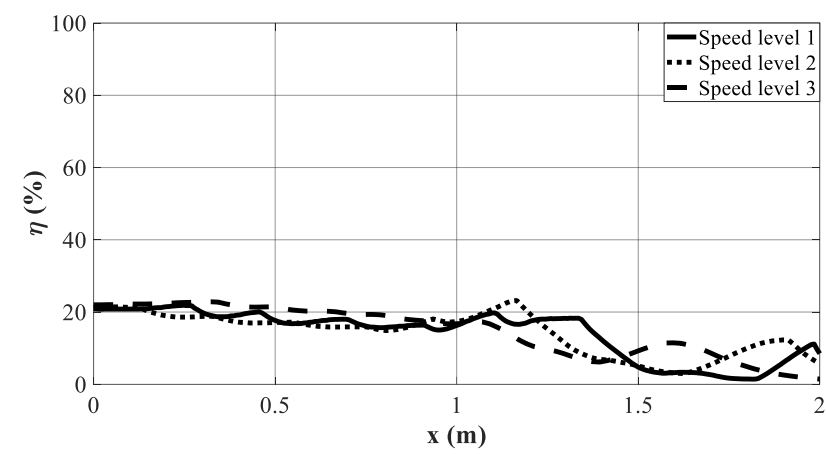

Figure. 11 Power transfer efficiency at gap $0.20 \mathrm{~m}$

\subsubsection{The gap is $0.20 \mathrm{~m}$}

Comparison of dynamic wireless power transfer efficiency at gap 0.20 meter when the speed changes are shown in Fig. 11, it was found that the speed level 2 gave the highest power transfer efficiency $23.19 \%$. The power transfer efficiency value has a very different. It makes the average power transfer efficiency lower than the speed level 3. The speed level 3 has an average power transfer efficiency of $14.67 \%$. At the speed level 3 is the fastest speed so the power transfer efficiency is smoother, resulting in more continuity than other speed ratings and more average power transfer efficiency. At all speed levels, the power transfer efficiency values were nearby resulting in similar power transfer efficiency values in the beginning of the experiment. In this experiment, the gap was the largest resulting in less power transfer efficiency in the system. In some periods of the experiment, the power transfer efficiency was reduced and turned to be highly efficient again because the power receiver sector may be unable to receive power from this power transmission sector but when it moves over the power transmission sector it can receive power from the next power transmission sector. Although the power transfer efficiency when the speed level changes are different. They are still considered similar so indicating that the speed has a low impact on the power transfer efficiency.

\subsection{Comparison results in case of adjusting the speed}

\subsubsection{Speed level 1}

Comparison of dynamic wireless power transfer efficiency at speed level 1 when the gap changes are shown in Fig. 12, It was found that at gap 0.10 meter gave the highest power transfer efficiency of $76.86 \%$ and gap 0.20 meter gave the lowest power transfer efficiency $1.42 \%$. The big gap gave the lower power transfer efficiency. It shows that the gap has a 


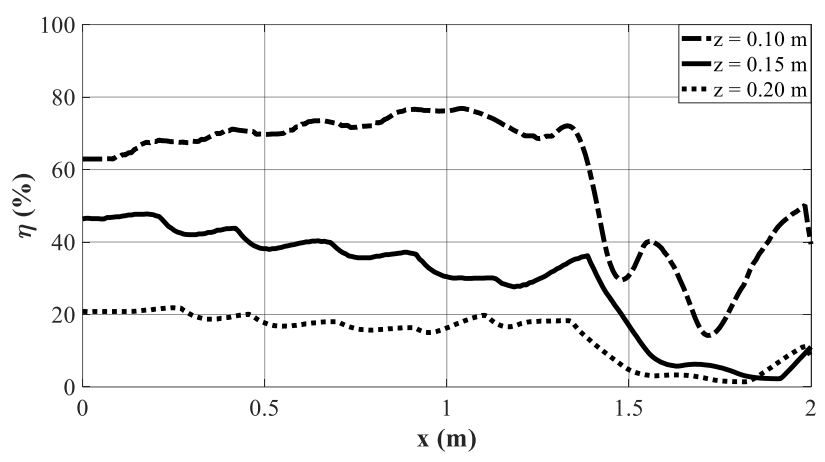

Figure. 12 Power transfer efficiency at speed level 1

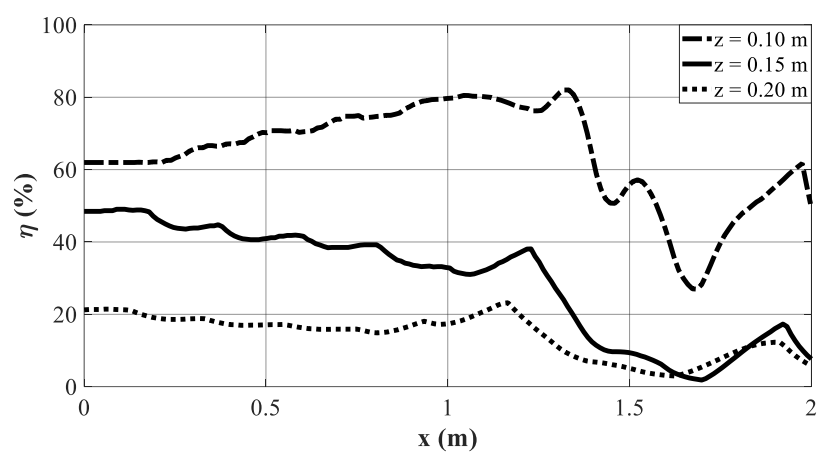

Figure. 13 Power transfer efficiency at speed level 2

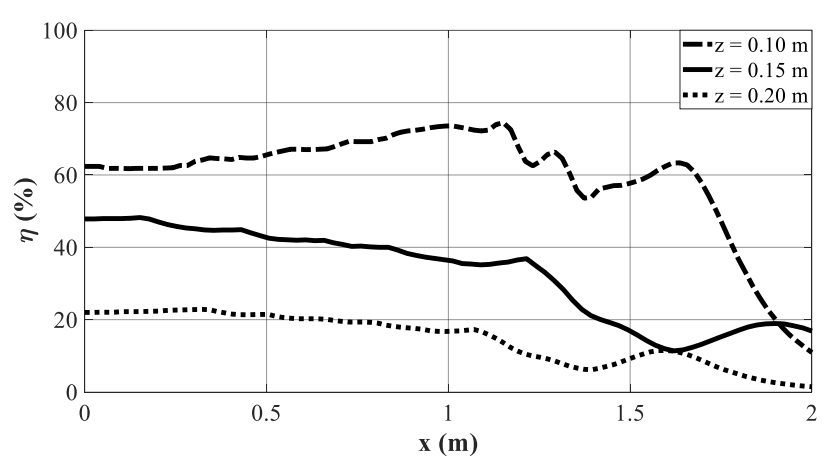

Figure. 14 Power transfer efficiency at speed level 3

Table 3. Power transfer efficiency

\begin{tabular}{|c|c|c|c|c|}
\hline \multirow{2}{*}{$\begin{array}{c}\text { Gap } \\
(\mathbf{m})\end{array}$} & \multirow{2}{*}{$\begin{array}{c}\text { Speed } \\
\text { level }\end{array}$} & \multicolumn{3}{|c|}{ Efficiency (\%) } \\
\cline { 3 - 5 } 0 & & Maximum & Minimum & Average \\
\hline \multirow{3}{*}{0.10} & 1 & 76.86 & 14.20 & 59.47 \\
\cline { 2 - 5 } & 2 & 82.01 & 26.96 & 64.62 \\
\hline \multirow{3}{*}{0.15} & 3 & 74.44 & 10.91 & 59.68 \\
\cline { 2 - 5 } & 2 & 47.74 & 2.26 & 29.23 \\
\cline { 2 - 5 } & 3 & 49.03 & 2.83 & 29.32 \\
\hline \multirow{3}{*}{0.20} & 1 & 14.22 & 11.37 & 32.92 \\
\cline { 2 - 5 } & 2 & 14.19 & 1.42 & 14.17 \\
\cline { 2 - 5 } & 3 & 14.67 & 1.94 & 14.19 \\
\hline
\end{tabular}

significant effect on the power transfer efficiency.

\subsubsection{Speed level 2}

Comparison of dynamic wireless power transfer efficiency at speed level 2 when the gap changes are shown in Fig. 13, It was found that at gap 0.10 meter gave the highest power transfer efficiency of $82.01 \%$ and gap 0.15 meter gave the lowest power transfer efficiency $2.83 \%$. The big gap gave the lower power transfer efficiency. It shows that the gap has a significant effect on the power transfer efficiency.

\subsubsection{Speed level 3}

Comparison of dynamic wireless power transfer efficiency at speed level 3 when the gap changes are shown in Fig. 14, It was found that at gap 0.10 meter gave the highest power transfer efficiency of $74.44 \%$ and gap 0.20 meter gave the lowest power transfer efficiency $1.44 \%$. The big gap gave the lower power transfer efficiency. It shows that the gap has a significant effect on the power transfer efficiency. The results of the experiment can be summarized as shown in Table 3.

\section{Conclusion}

This paper presents a dynamic wireless power transfer system consists of multiple power transmission sectors in parallel with a single inverter circuit for light duty electric vehicles. The dynamic wireless power transfer provides a maximum power transfer efficiency is $82.01 \%$ and an average power transfer efficiency is $64.62 \%$ at a gap of $0.10 \mathrm{~m}$ and speed level 2. As the gap increases, the power transfer efficiency decreases. where the gap is very high, it may cause the power transfer efficiency to decrease at some point and return to high efficiency again because the power receiver sector cannot receive power from the power transmission sector but when electric vehicles move over the power transmission sector, the power receiver sector can receive power from the next set of power transmission sectors. This shows that the gap greatly affects the power transfer efficiency. However, the speed increased does not make a huge difference in power transfer efficiency, but the power transfer efficiency value is smoother. Although the power transfer efficiency values at the speed level changes are different, they are still considered similar, indicating that the speed has a low effect on the power transfer efficiency.

\section{Conflicts of interest (Mandatory)}

The authors declare no conflict of interest.

\section{Author contributions (Mandatory)}

conceptualization, PHARIDA; methodology, PHARIDA and CHAIYUT; validation, PHARIDA; formal analysis, PHARIDA and CHAIYUT; investigation, PHUMIN; resources, PHARIDA; data curation, PHARIDA; writing - original draft 
preparation, PHARIDA; writing - review and editing, CHAIYUT and PHUMIN; visualization, PHARIDA; supervision, CHAIYUT.

\section{References}

[1] L. Sun, D. Ma, and H. Tang, "A Review of Recent Trends in Wireless Power Transfer Technology and Its Applications in Electric Vehicle Wireless Charging", International Journal of Renewable and Sustainable Energy Reviews, Vol. 91, pp. 490-503, 2018.

[2] Y. J. Jang, "Survey of the Operation and System Study on Wireless Charging Electric Vehicle Systems", International Journal of Transportation Research Part C: Emerging technologies, Vol. 95, pp. 844-866, 2018.

[3] C. Panchal, S. Stegen, and J. Lu, "Review of static and dynamic wireless electric vehicle charging system", International Journal of Engineering Science and Technology, Vol. 21, No. 5, pp. 922-937, 2018.

[4] X. Zhang, Z. Yuan, Q. Yang, Y. Li, J. Zhu, and Y. Li, "Coil Design and Efficiency Analysis for Dynamic Wireless Charging System for Electric Vehicles", International Journal of IEEE Transactions on Magnetics, Vol. 52, No. 7, 2016.

[5] L. Shuguang, Y. Zhenxing, and L. Wenbin, "Electric vehicle dynamic wireless charging technology based on multi-parallel primary coils", In: Proc. of the IEEE International Conf. on Electronics and Communication Engineering, Xi'an, China, pp. 120-124, 2018.

[6] C. Anyapo, N. Teerakawanich, and C. Mitsantisuk, "Development of multi-coils fullbridge resonant inverter for dynamic wireless power transfer", In: Proc. of the 14th International Conf. on Electrical Engineering/Electronics, Computer, Telecommunications and Information Technology, Phuket, Thailand, pp. 588-591, 2017.

[7] X. Dai, J. C. Jiang, and J. Q. Wu, "Charging area determining and power enhancement method for multiexcitation unit configuration of wirelessly dynamic charging EV system", International Journal of IEEE Transactions on Industrial Electronics, Vol. 66, No. 5, pp. 4086-4096, 2019.

[8] S. Jeong, Y. J. Jang, D. Kum, and M. S. Lee, "Charging automation for electric vehicles: Is a smaller battery good for the wireless charging electric vehicles?", International Journal of IEEE Transactions on Automation Science and Engineering, Vol. 16, No. 1, pp. 486-497, 2019.
[9] Z. Bi, G. A. Keoleian, Z. Lin, M. R. Moore, K. Chen, L. Song, and Z. Zhao, "Life Cycle Assessment and Tempo-spatial Optimization of Deploying Dynamic Wireless Charging Technology for Electric Cars", International Journal of Transportation Research Part C: Emerging Technologies, Vol. 100, pp. 53-67, 2019.

[10] K. H. Yi, J. Y. Jung, B. H. Lee, and Y. S. You, "Study on a Capacitive Coupling Wireless Power Transfer with Electric Vehicle's Dielectric Substrates for Charging an Electric Vehicle", In: Proc. of 2017 19th European Power Electronics and Drives Association, Warsaw, Poland, pp. 1-7, 2017.

[11] Y. Guo, L. Wang, Q. Zhu, C. Liao, and F. Li, "Switch-On Modeling and Analysis of Dynamic Wireless Charging System Used for Electric Vehicle", International Journal of IEEE Transactions on Industrial Electronics, Vol. 63, No. 10, pp. 6568-6579, 2016.

[12] A. T. Cabrera, J. A. A. Sánchez, M. Longo, and F. Foiadelli, "Sensitivity Analysis of a Bidirectional Wireless Charger for EV", In: Proc. of 2016 IEEE International Conference on Renewable Energy Research and Applications, Birmingham, UK, pp. 1113-1116, 2016.

[13] H. Feng, T. Cai, S. Duan, J. Zhao, X. Zhang, and C. Chen, "An LCC-Compensated Resonant Converter Optimized for Robust Reaction to Large Coupling Variation in Dynamic Wireless Power Transfer", International Journal of IEEE Transactions on Industrial Electronics, Vol. 63, No. 10, pp. 6591-6601, 2016.

[14] A. Poorfakhraei, G. Movaghar, and F. Tahami, "Optimum design of coils in a dynamic wireless electric vehicle charger with misalignment compensation capability", In: Proc. of the 8th Power Electronics, Drive Systems \& Technologies Conf., Mashhad, Iran, pp. 419-424, 2017.

[15] J. Lee and B. Han, "A Bidirectional Wireless Power Transfer EV Charger Using SelfResonant PWM", International Journal of IEEE Transactions on Power Electronics, Vol. 30, No. 4, pp. 1784-1787, 2015.

[16] X. Liu and G. Wang, "A Novel Wireless Power Transfer System with Double Intermediate Resonant Coils", International Journal of IEEE Transactions on Industrial Electronics, Vol. 63, No. 4, pp. 2174-2180, 2016.

[17] X. Qu, H. Han, S. Wong, C. K. Tse, and W. Chen, "Hybrid IPT Topologies with Constant Current or Constant Voltage Output for Battery Charging Applications", International Journal 
of IEEE Transactions on Power Electronics, Vol. 30, No. 11, pp. 6329-6337, 2015.

[18] Z. Li, C. Zhu, J. Jiang, K. Song, and G. Wei, “A 3-kW Wireless Power Transfer System for Sightseeing Car Supercapacitor Charge", International Journal of IEEE Transactions on Power Electronics, Vol. 32, No. 5, pp. 33013316, 2017. 